Research Article

\title{
Labyrinthine Structure with Subwavelength and Broadband Sound Insulation
}

\author{
Heng Jiang,, ${ }^{1,2}$ Yu Liu, ${ }^{1,2}$ Wenshuai Xu, ${ }^{1,2}$ Tao Yang, ${ }^{1,2}$ Dongliang Pei, ${ }^{1,2}$ Meng Chen $\mathbb{D}^{1,2}$ \\ and Yuren Wang $\mathbb{D}^{1,2}$
}

${ }^{1}$ Key Laboratory of Microgravity, Institute of Mechanics, Chinese Academy of Sciences, Beijing 100190, China
${ }^{2}$ University of Chinese Academy of Sciences, Beijing 100049, China

Correspondence should be addressed to Meng Chen; chenmeng@imech.ac.cn and Yuren Wang; yurenwang@imech.ac.cn

Received 13 July 2020; Revised 1 November 2020; Accepted 4 November 2020; Published 12 November 2020

Academic Editor: Jinqiang Gan

Copyright (C) 2020 Heng Jiang et al. This is an open access article distributed under the Creative Commons Attribution License, which permits unrestricted use, distribution, and reproduction in any medium, provided the original work is properly cited.

In this text, the combination of spiral structure and zigzag channels is introduced to design labyrinthine structures, in which sound waves can propagate alternately in the clockwise and counterclockwise directions. Finite element method and S-parameter retrieval method are used to calculate band structures, effective parameters, and transmission properties of the structures. The influences of different structural parameters on their acoustic properties are also studied. These results show labyrinthine structures have multiple bandgaps in the range of $0 \mathrm{~Hz}-1000 \mathrm{~Hz}$, and the proportion of bandgaps exceeds $33 \%$, which indicates labyrinthine structures have good broadband properties. The normalized frequency of the lowest bandgaps is far smaller than 1 , which indicates the structures take good control of sound waves on subwavelength scale. Combining units with different structural parameters can achieve better sound insulation. This research provides a new kind of space-coiling structure for low-frequency and broadband sound waves control, which have excellent application prospects.

\section{Introduction}

Noise is ubiquitous in the modern world and causes considerable trouble in daily life, particularly in the range of $0 \mathrm{~Hz}-1000 \mathrm{~Hz}$ [1]. In order to control the noise effectively, a very large scale structure is usually needed according to the mass density law, but this is not practical for engineering applications. Therefore, controlling low-frequency noise remains a challenging problem.

Locally resonant acoustic metamaterials provide a new way to control sound waves. These materials can break the restrictions of the mass density law and cause low-frequency sound waves to be attenuated effectively on the subwavelength scale, which show that these materials have promising application prospects [2-19]. To control sound waves effectively within the range of $0 \mathrm{~Hz}$ to $1000 \mathrm{~Hz}$ using the small size structural units, researchers have carried out extensive study into the acoustic properties of locally resonant acoustic metamaterials. Liu et al. prepared a locally resonant phononic crystal with negative mass density, and a
$2 \mathrm{~cm}$ thick sheet consisting of the unit attenuates the acoustic wave at $400 \mathrm{~Hz}$ effectively [2]. Yang et al. prepared a simpler structure composed of membrane acoustic metamaterials. Within $100 \mathrm{~Hz}-1000 \mathrm{~Hz}$, this structure can break the mass density law by 200 times and realize total reflection at the antiresonance frequency. By coupling membrane acoustic metamaterials with different resonance frequencies, it is also possible to realize broadband acoustic control $[8,9]$. Using finite element calculations and impedance tube testing, Naify et al. carried out research into the influence of additional mass and membrane preloading on acoustic properties of membrane acoustic metamaterials and then designed an annular membrane acoustic metamaterial with broadband properties [12-14]. Membrane acoustic metamaterials can control sound waves in the range of $0 \mathrm{~Hz}-1000 \mathrm{~Hz}$, but the membrane thickness is very small and the rigidity of the structure is low. Due to those, any subtle change in material prestress will cause the change of resonance frequency, this leads to acoustic performance instability for the membrane acoustic metamaterial and increases 
the difficulty of controlling low-frequency sound waves. In addition, locally resonant acoustic metamaterials can only control low-frequency sound waves at the resonance frequency and the bandgap is also narrow. To realize broadband sound wave control, it is necessary to couple resonant units with different resonance frequencies, which increases the structural complexity.

To achieve better control of low-frequency and broadband acoustic waves, researchers introduce the space-coiling method into acoustic metamaterials and design acoustic metamaterials with high refractive indexes, multiple vibration modes, and extraordinary acoustic properties, thus providing a new method to achieve broadband control of acoustic waves with a single structural unit [20-36]. The extraordinary properties of these space-coiling acoustic metamaterials have attracted extensive research attention. A series of studies have been carried out on sound insulation using space-coiling acoustic metamaterials in low- and broadband frequency ranges. Cheng et al. proposed a spacecoiling metasurface with a high refractive index based on the Mie-resonance properties, which can realize total reflection of low-frequency sound waves [25]. Man et al. introduced self-similar fractal theory into the design of space-coiling acoustic metamaterials and prepared self-similar fractal acoustic metamaterials, which can control low-frequency and broadband sound waves easily [27]. Song et al. prepared a Hilbert fractal structure and performed theoretical calculations and experimental tests on this structure. They found that their structure could attenuate sound waves effectively in the $225 \mathrm{~Hz}-1175 \mathrm{~Hz}$ frequency range, thus effectively controlling the low-frequency sound waves [31]. These results show that space-coiling structures can achieve better control of low-frequency and broadband sound waves in $0 \mathrm{~Hz}-1000 \mathrm{~Hz}$ compared with locally resonant acoustic metamaterials. At present, researchers mainly use zigzag channels, self-similar fractal structures, and spiral structures to design the space-coiling structures. The results show different metamaterials with a variety of extraordinary acoustic properties. However, there has been little research to date on a combination of different structures.

In this paper, the combination of spiral structure and zigzag channels is introduced to design labyrinthine structures. The labyrinthine structures with different numbers of folds are built up and their bandgaps and transmission properties are studied. The results show the new designed labyrinthine structures contain more bandgaps than previous designs and they can also realize better control of the low-frequency and broadband sound waves in $0 \mathrm{~Hz}$ to $1000 \mathrm{~Hz}$.

\section{Structural Design}

In contrast to traditional zigzag channels, self-similar fractal structures, and spiral structures, we introduce a combination of spiral structure and zigzag channels into the unit design, in which sound waves can propagate clockwise and counterclockwise alternately. To explore the effects of the transmission path on the acoustic properties of the labyrinthine structure, labyrinthine structural units with four, six, and eight time folds are designed, as illustrated in Figures $1(\mathrm{a})-1(\mathrm{c})$, where $a(a=72 \mathrm{~mm})$ is the lattice constant of the labyrinthine structural unit, $d(d=3 \mathrm{~mm})$ is the width of the transmission channel, $t(t=1 \mathrm{~mm})$ is the thickness of the diaphragm, and the arrow indicates the sound wave transmission direction. To explore the effects of the acoustic channel width on the acoustic properties of the labyrinthine structure, six-times-folded labyrinthine structure units with acoustic channel widths of $2 \mathrm{~mm}, 4 \mathrm{~mm}$, and $5 \mathrm{~mm}$ are designed, as shown in Figure 1(d)-1(f). The figure shows that the acoustic wave will rotate multiple times in the structure and that its transmission path is several times longer than the straight-line path; this causes the labyrinthine structure have a high equivalent refractive index, meaning that the structure has extraordinary acoustic properties.

\section{Band Structure Calculations}

To enable further study of the effects of the structural parameters on the acoustic properties, such as the number of folds and the width of acoustic channels, finite element method and Comsol software are adopted to calculate the band structure of the labyrinthine structures. The individual units are arranged in space in the form of a square lattice structure, and the lattice cell size is $a_{1}\left(a_{1}=76 \mathrm{~mm}\right)$. The Floquet-Bloch periodic boundary conditions are applied at the boundaries of the structural units. The wave vector $\boldsymbol{k}$ is scanned along the irreducible Brillouin zone, and the values of the characteristic frequency $w$ corresponding to different wave vectors $\boldsymbol{k}$ are obtained. Thus, the dispersion relation $\boldsymbol{w}(\boldsymbol{k})$ can be used to represent the band structure, and the results of the calculations are shown in Figure 2.

Figure 2(a) shows the calculation results of the fourtimes-folded labyrinthine acoustic metamaterial. The figure shows that the structure has four-omnidirectional bandgaps, which are [228.95 Hz-325.65 Hz], [353.34 Hz-491.11 Hz], [628.03 Hz-729.52 Hz], and [906.78 Hz-937.53 Hz]. In the frequency range of $0 \mathrm{~Hz}-1000 \mathrm{~Hz}$, the omnidirectional bandgaps account for $36.67 \%$ of the total range. Figure 2 (b) shows the calculation results of the six-times-folded labyrinthine acoustic metamaterial. The figure shows that there are five omnidirectional bandgaps present in the structure, which are [186.92 Hz-273.06 Hz], [313.12 Hz-371.42 Hz], $[455.64 \mathrm{~Hz}-609.95 \mathrm{~Hz}],[622.2 \mathrm{~Hz}-651.9 \mathrm{~Hz}]$, and $[830.67$ $\mathrm{Hz}-878.05 \mathrm{~Hz}$ ]. In the frequency range of $0 \mathrm{~Hz}-1000 \mathrm{~Hz}$, the omnidirectional bandgaps account for $37.58 \%$ of the total range. Figure 2(c) shows the calculation results of the eighttimes-folded labyrinthine acoustic metamaterial. The figure shows that there are six omnidirectional bandgaps in this structure, which are $[151.77 \mathrm{~Hz}-248.97 \mathrm{~Hz}],[290.05$ $\mathrm{Hz}-301.82 \mathrm{~Hz}$, [438.95 Hz-527.23 Hz], [549.4 Hz-582.12 $\mathrm{Hz}$ ], [704.21 Hz-788.46 Hz], and [830.75 Hz-850.3 Hz]. In the frequency range of $0 \mathrm{~Hz}-1000 \mathrm{~Hz}$, the omnidirectional bandgaps account for $33.38 \%$ of the total range. These results show that, as the number of folds increases, causing the effective transmission path increases, the frequency range of the lowest bandgap gradually decreases in tandem, and the structure will then exhibit better low-frequency properties. 


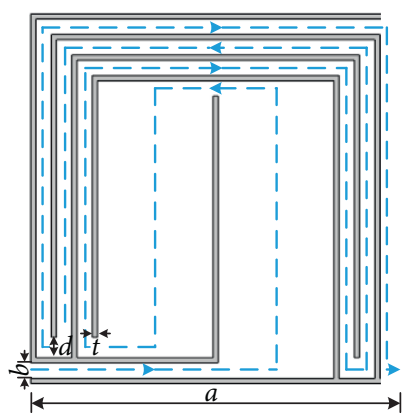

(a)

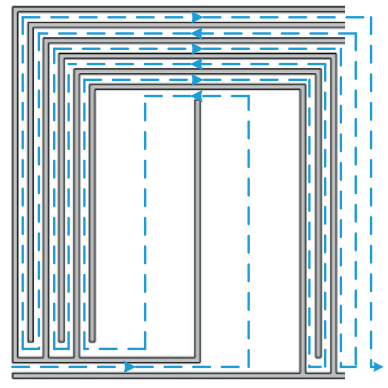

(d)

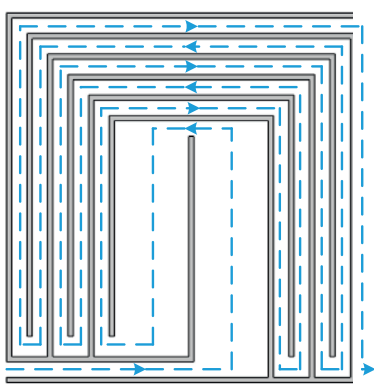

(b)

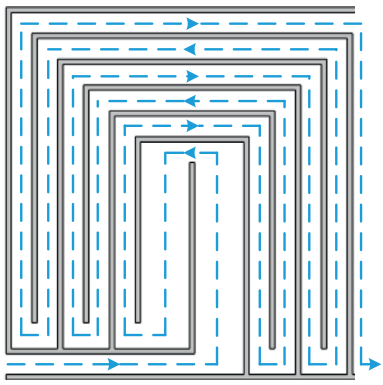

(e)

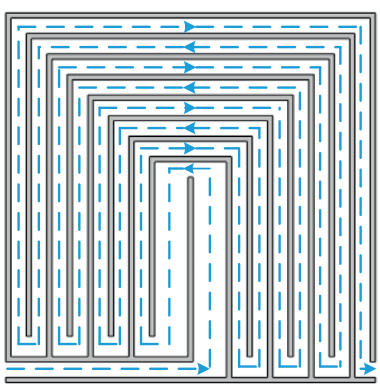

(c)

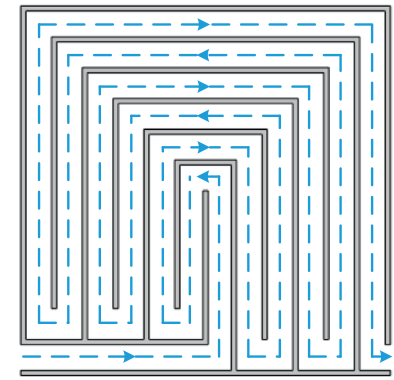

(f)

FIGURE 1: Labyrinthine structure units. (a) Four-times-folded labyrinthine structure unit. (b) Six-times-folded labyrinthine structure unit. (c) Eight-times-folded labyrinthine structure unit. (d) Six-times-folded labyrinthine structural unit with sound channel width of $2 \mathrm{~mm}$. (e) Six-times-folded labyrinthine structural unit with sound channel width of $4 \mathrm{~mm}$. (f) Six-times-folded labyrinthine structural unit with sound channel width of $5 \mathrm{~mm}$.

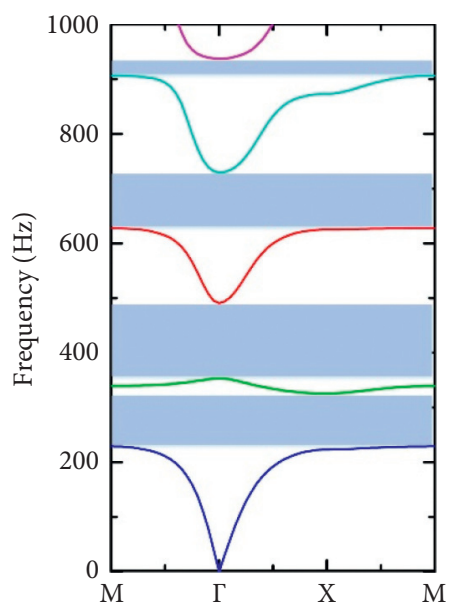

(a)

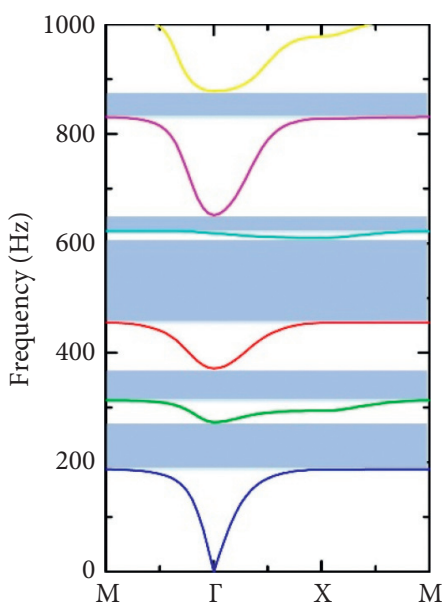

(b)

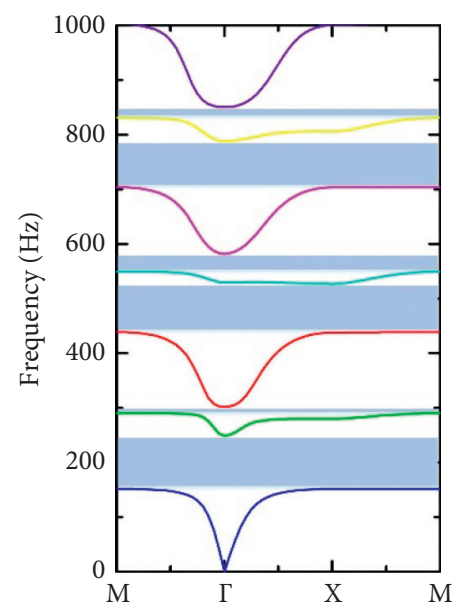

(c)

Figure 2: Continued. 


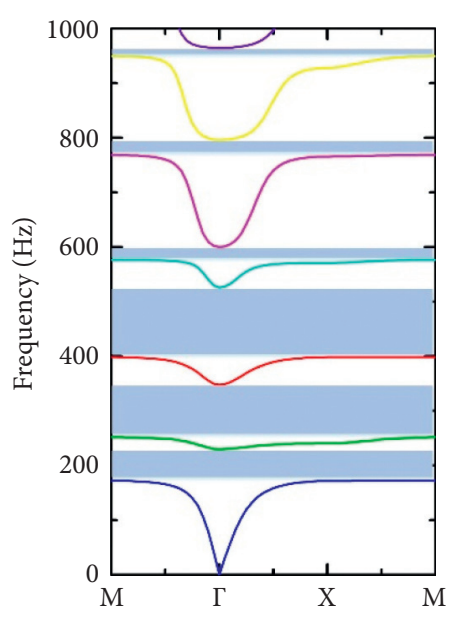

(d)

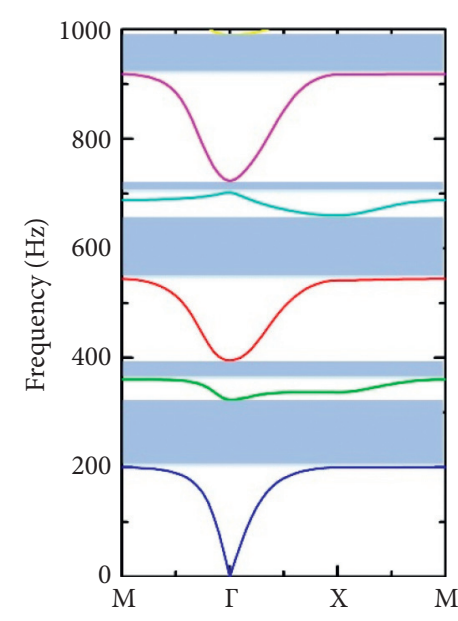

(e)

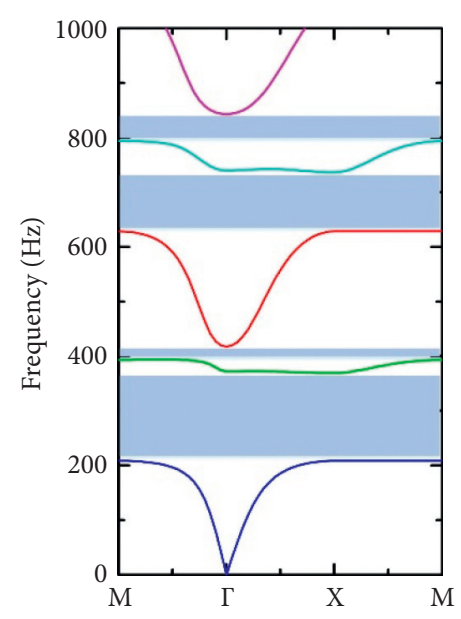

(f)

Figure 2: (a)-(c) Four-to eight-times-folded labyrinthine acoustic metamaterial band structures. (d)-(f) Six-times-folded labyrinthine acoustic metamaterial band structures with sound channel widths of $2 \mathrm{~mm}, 4 \mathrm{~mm}$, and $5 \mathrm{~mm}$. The omnidirectional bandgaps of the structures are highlighted using light blue shading.

In the eight-times-folded labyrinthine structure in particular, the normalized frequency $\left(f a_{1} / c\right)$ is within the range of $0.034-0.056$, which is far less than 1 , thus indicating that the labyrinthine structure can achieve better subwavelength sound wave control. In addition, the omnidirectional bandgaps of the labyrinthine structures with different numbers of folds account for more than $33 \%$ of the frequency range from $0 \mathrm{~Hz}-1000 \mathrm{~Hz}$, which has higher proportion than mentioned in the space-coiling acoustic metamaterials built with zigzag channels and Hilbert fractal structures, particularly in the case of the six-times-folded labyrinthine structure. Its omnidirectional bandgaps account for $37.58 \%$ of the frequency of interest, indicating that this labyrinthine structure has good broadband sound insulation properties.

Figure 2(d) shows the calculation results for the sixtimes-folded labyrinthine acoustic metamaterial with the sound channel width of $2 \mathrm{~mm}$. There are six omnidirectional bandgaps in this structure, which are $[172.18 \mathrm{~Hz}-229.91 \mathrm{~Hz}]$, [251.5 Hz-347.53 Hz], [398.13 Hz-525.86 Hz], $[576.61 \mathrm{~Hz}-$ $600.27 \mathrm{~Hz}], \quad[768.36 \mathrm{~Hz}-796.17 \mathrm{~Hz}]$, and $[949.29 \mathrm{~Hz}-$ $964.22 \mathrm{~Hz}$. In the frequency range of $0 \mathrm{~Hz}-1000 \mathrm{~Hz}$, the omnidirectional bandgaps account for $34.79 \%$ of the range. Figure 2(e) shows the calculation results for the six-timesfolded labyrinthine acoustic metamaterial with the sound channel width of $4 \mathrm{~mm}$. There are five omnidirectional bandgaps in this structure, which are $[199.73 \mathrm{~Hz}-323.38 \mathrm{~Hz}]$, $[360.24 \mathrm{~Hz}-395.1 \mathrm{~Hz}], \quad[544.2 \mathrm{~Hz}-659.86 \mathrm{~Hz}], \quad[702.75 \mathrm{~Hz}-$ $722.78 \mathrm{~Hz}]$, and $[918.11 \mathrm{~Hz}-989.55 \mathrm{~Hz}]$. In the frequency range of $0 \mathrm{~Hz}-1000 \mathrm{~Hz}$, the omnidirectional bandgaps account for $36.56 \%$ of the range. Figure $2(\mathrm{f})$ presents the calculation results for the six-times-folded labyrinthine acoustic metamaterial with the sound channel width of $5 \mathrm{~mm}$. There are four omnidirectional bandgaps in this structure, which are $[208.59 \mathrm{~Hz}-368.87 \mathrm{~Hz}],[392.94 \mathrm{~Hz}-$ $417.48 \mathrm{~Hz}],[628.56 \mathrm{~Hz}-736.45 \mathrm{~Hz}]$, and $[793.59 \mathrm{~Hz}-842.87$
$\mathrm{Hz}$. In the frequency range of $0 \mathrm{~Hz}-1000 \mathrm{~Hz}$, the omnidirectional bandgaps account for $34.20 \%$ of the range. These results indicate that, as the acoustic channel width narrows, the frequency range of the lowest bandgap decreases gradually and the six-times-folded labyrinthine acoustic metamaterials with different acoustic channel widths show good low-frequency and broadband sound insulation properties. The research results above show that adjustment of the structural parameters, including the number of folds and width of the acoustic channel, modulates the bandgap frequency range of the structure effectively. The proposed labyrinthine structure possesses good low-frequency and broadband acoustic properties, which means it has bright application prospects for the subwavelength acoustic wave control.

\section{Calculation of Equivalent Parameters}

As part of the calculation of the band structure, based on a six-times-folded labyrinthine structure with the sound channel width of $3 \mathrm{~mm}$, the effects of different numbers of folds and sound channel widths on the acoustic properties are studied. To enable further exploration of the bandgap generation mechanism of the labyrinthine structure, the effective mass density $\rho_{\text {eff }}$ and the effective bulk modulus $B_{\text {eff }}$ of the structure are calculated using the S-parameter retrieval method [37], and the expressions are as follows:

$$
\begin{aligned}
& \rho_{\text {eff }}=\varepsilon \times n, \\
& B_{\text {eff }}=\frac{\varepsilon}{n},
\end{aligned}
$$

where $\varepsilon$ is the equivalent impedance and $n$ is the effective refractive index, and these parameters can be calculated as follows: 

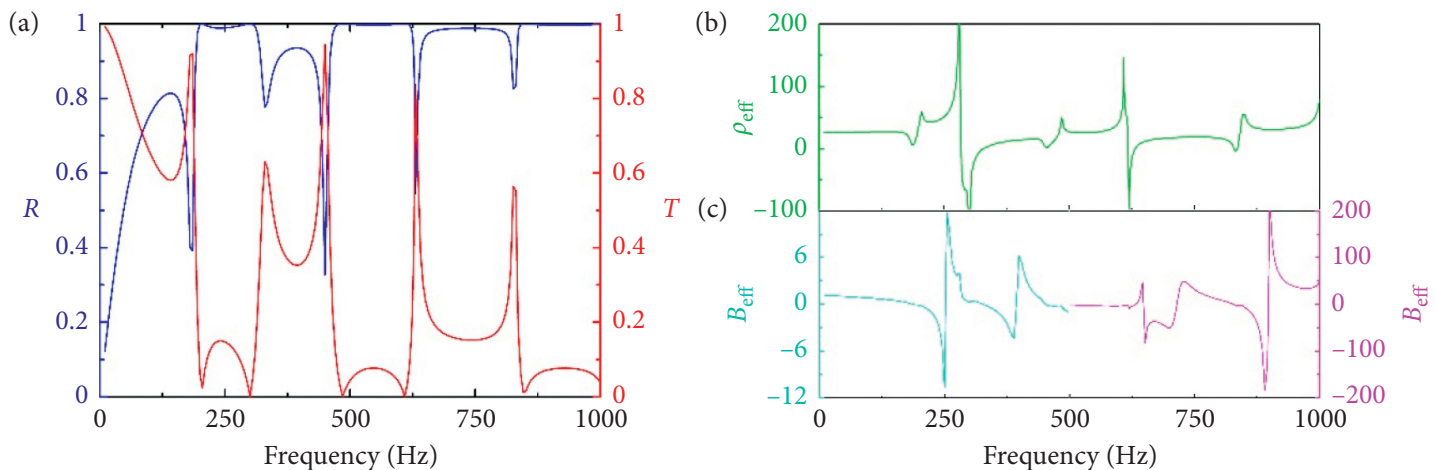

Figure 3: (a) Calculation results for the transmission coefficient. (b) Calculation results for the effective mass density. (c) Calculation results for the effective bulk modulus.

$$
\begin{aligned}
& \varepsilon=\frac{\mp \sqrt{\left(R^{2}-T^{2}-1\right)^{2}-4 T^{2}}}{1-2 R+R^{2}-T^{2}}, \\
& n=\frac{-i \log \left(\left(\left(1-R^{2}+T^{2}+r\right)\right) / 2 T\right)+2 \pi m}{k d},
\end{aligned}
$$

where $R$ is the reflection coefficient and $T$ is the transmission coefficient. Finite element method is used to calculate the transmission and reflection coefficients of the structure, and the calculated results are shown in Figure 3(a). By introducing these transmission and reflection coefficients into equations (3) and (4), the equivalent impedance and the effective refractive index of the structure can then be calculated. Subsequently, solving equations (1) and (2) simultaneously, the effective mass density and the effective bulk modulus of the structure can be obtained, with results as shown in Figures 3(b) and 3(c), respectively. The calculation results show that the effective bulk modulus of the structure is negative within the frequency ranges of $[190 \mathrm{~Hz}-250 \mathrm{~Hz}], \quad[330 \mathrm{~Hz}-390 \mathrm{~Hz}], \quad[455 \mathrm{~Hz}-630 \mathrm{~Hz}]$, $[651 \mathrm{~Hz}-711 \mathrm{~Hz}]$, and $[831 \mathrm{~Hz}-896 \mathrm{~Hz}]$; similarly, in the frequency ranges of $[285 \mathrm{~Hz}-325 \mathrm{~Hz}]$ and $[619 \mathrm{~Hz}-631 \mathrm{~Hz}]$, the effective mass density of the structure is also negative. The labyrinthine structures have multiple negative effective parameter frequency ranges, which are the reason why the structures have multiple bandgaps.

\section{Calculation of Transmission Properties}

The calculation results for band structure and effective parameters indicate that the labyrinthine structures have good low-frequency and broadband properties. To study the acoustic properties of these structures further, transmission properties of labyrinthine structural units for different numbers of folds and acoustic channel widths are calculated. Transmission properties of three identical structural units are calculated firstly, and results are shown in Figure 4. There are multiple transmission loss peaks in the $0 \mathrm{~Hz}-1000 \mathrm{~Hz}$ frequency range, which agree well with frequency ranges of bandgaps. The calculation results also show that the positions of transmission loss peaks vary with the adjustment of structural parameters, including the number of folds and

width of the acoustic channels. To achieve better broadband sound insulation, structural units with different numbers of folds and sound channel widths are combined and their transmission properties are obtained, which are shown in Figures 5 and 6.

Figure 5(a) shows the transmission loss of four- and eight-times-folded labyrinthine structure units. This result shows labyrinthine structures provide good broadband sound insulation performance in the frequency ranges of $[150 \mathrm{~Hz}-350 \mathrm{~Hz}],[375 \mathrm{~Hz}-655 \mathrm{~Hz}]$, and $[675 \mathrm{~Hz}-845 \mathrm{~Hz}]$. When compared with structural units with identical structural units, the superposition of structural units can widen the frequency range of the transmission loss peaks effectively and achieve better broadband sound insulation. Figures 5(b)-5(d) show the calculated sound pressure level distributions of these structural units at $190 \mathrm{~Hz}, 500 \mathrm{~Hz}$, and $800 \mathrm{~Hz}$, respectively. It can be seen from these figures that the structures can achieve $60 \mathrm{~dB}, 38.5 \mathrm{~dB}$, and $53 \mathrm{~dB}$ attenuation at corresponding frequencies.

Figure 6(a) shows the transmission losses of the structural units with sound channel widths of $3 \mathrm{~mm}-5 \mathrm{~mm}$ superimposed. In this structure, the transmission loss exceeds $10 \mathrm{~dB}$. In the frequency range of $0 \mathrm{~Hz}-1000 \mathrm{~Hz}$, the proportion of the transmission loss peak can reach $65 \%$. This structure can achieve better broadband sound insulation. In addition, compared with the structure with identical width, the method of superimposing structural units with different sound channel widths can broaden the frequency ranges of the transmission loss peak effectively. Figures 5(b)-5(d) show the calculated sound pressure level distributions of these structural units at $195 \mathrm{~Hz}, 555 \mathrm{~Hz}$, and $835 \mathrm{~Hz}$, respectively. The figures show that these structures can achieve attenuation of $49 \mathrm{~dB}, 60 \mathrm{~dB}$, and $47 \mathrm{~dB}$ at corresponding frequencies.

The calculated results for transmission properties above show labyrinthine structures have broadband sound insulation properties. By superimposing structural elements with different parameters, the frequency ranges of the peak transmission loss can be widened effectively and better broadband sound wave control can be achieved. In addition, the normalized frequency at the first transmission loss peak for all structural units is less than 0.056 , which is much smaller than 1, and the structure can also achieve better 


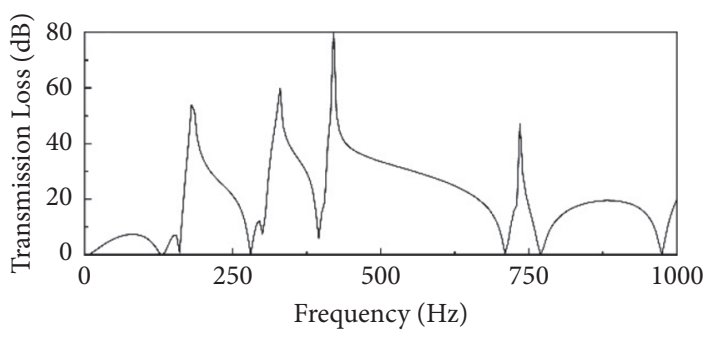

(a)

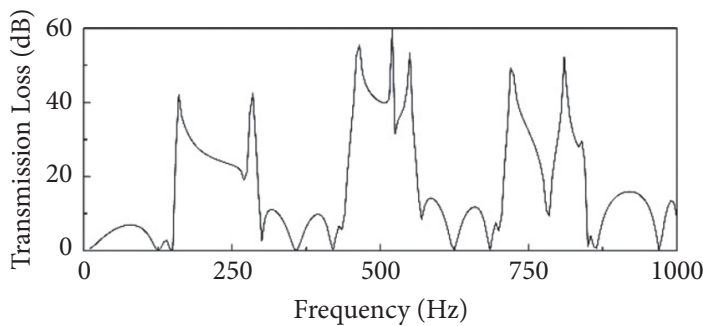

(c)

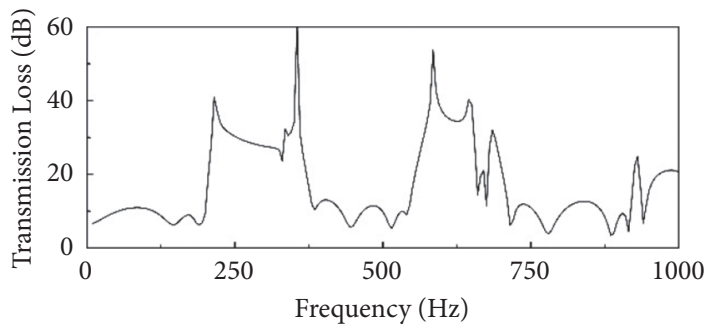

(e)

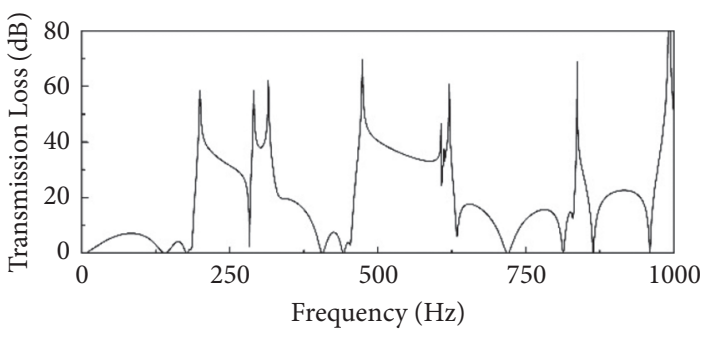

(b)

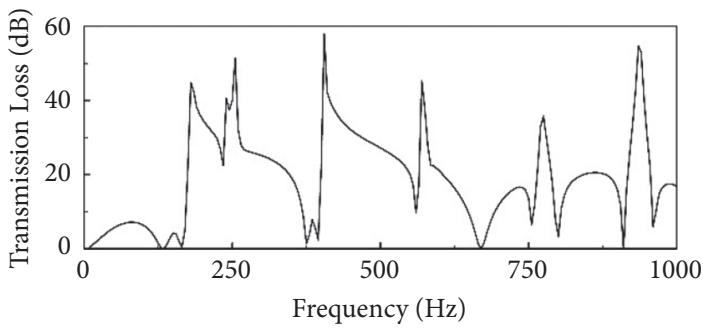

(d)

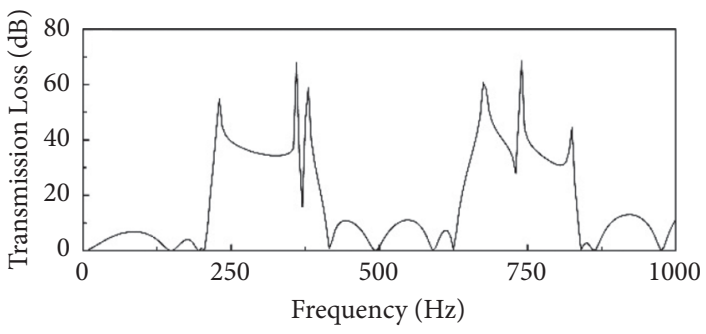

(f)

FIgUre 4: (a)-(c) Transmission losses of four-to eight-times-folded labyrinthine acoustic metamaterials. (d)-(f) Transmission losses of sixtimes-folded labyrinthine acoustic metamaterials with widths of $2 \mathrm{~mm}, 4 \mathrm{~mm}$, and $5 \mathrm{~mm}$.

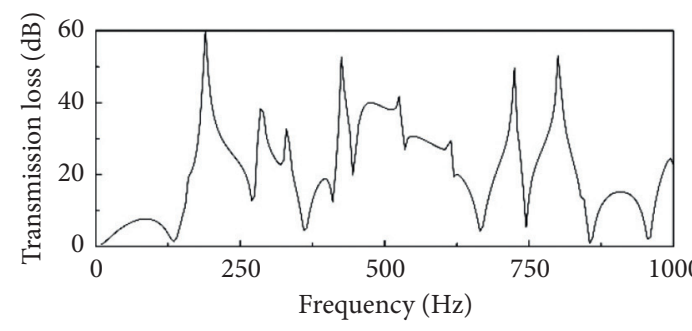

(a)

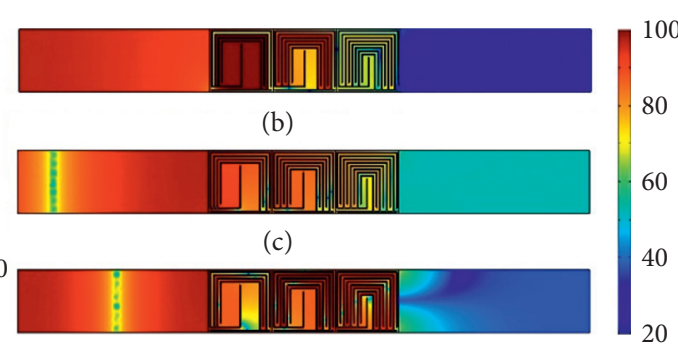

Sound pressure level $(\mathrm{dB})$

(d)

Figure 5: (a) Transmission loss of four-to eight-times-folded labyrinthine structure units. (b)-(d) Sound pressure level distributions at $190 \mathrm{~Hz}, 500 \mathrm{~Hz}$, and $800 \mathrm{~Hz}$, respectively.

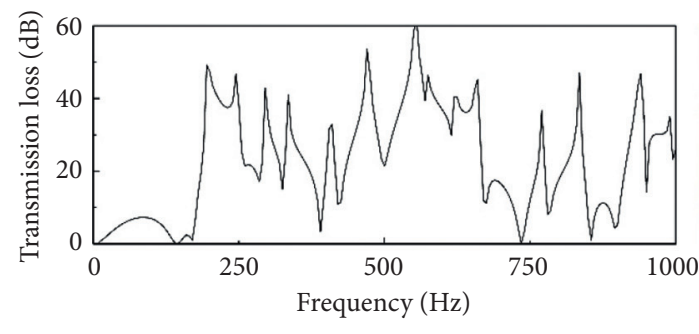

(a)

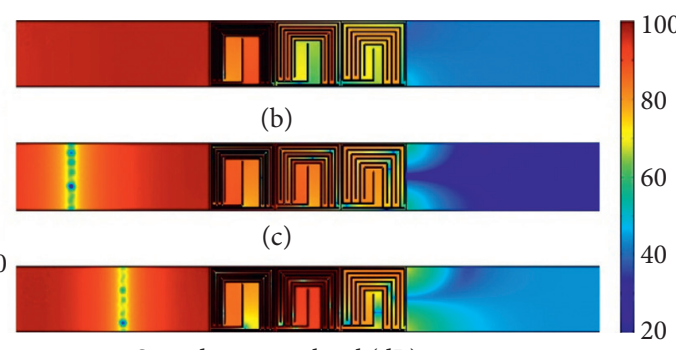

Sound pressure level $(\mathrm{dB})$

(d)

Figure 6: (a) Transmission loss of structural unit with sound channel widths of $3 \mathrm{~mm}-5 \mathrm{~mm}$ superimposed. (b)-(d) Sound pressure level distributions at $195 \mathrm{~Hz}, 555 \mathrm{~Hz}$, and $835 \mathrm{~Hz}$, respectively. 
subwavelength acoustic wave control. The labyrinthine structures have good low-frequency and broadband acoustic properties and provide a new kind of structure to achieve effective control of sound waves in the $0 \mathrm{~Hz}-1000 \mathrm{~Hz}$ frequency range.

\section{Conclusion}

In this paper, the combination of spiral structure and zigzag channels is introduced to design labyrinthine structures, in which sound waves can propagate clockwise and counterclockwise alternately. Excellent low-frequency and broadband properties in the $0 \mathrm{~Hz}-1000 \mathrm{~Hz}$ frequency range are shown through calculating and analyzing band structures, effective parameters, and transmission properties of these structures. These structures also have negative effective parameters in the frequency ranges of bandgaps, which are the reasons of the formation for these bandgaps. In these bandgaps, sound waves cannot propagate, thus producing multiple high transmission loss peaks. By superimposing structural units with different parameters, the frequency range of transmission loss peaks can be broadened effectively and better broadband acoustic wave control can be realized. In addition, the normalized frequencies of the first transmission loss peaks for all structural units are less than 0.056 , which are much smaller than 1 , meaning these structures can also achieve subwavelength sound waves control. These results presented above show labyrinthine structures have good low-frequency and broadband sound insulation properties, thus providing a new kind of space-coiling to achieve effective sound wave control in the $0 \mathrm{~Hz}-1000 \mathrm{~Hz}$ frequency range, which have bright prospects for use in practical applications.

\section{Data Availability}

The numerical data used to support the findings of this study are available from the corresponding author upon request.

\section{Conflicts of Interest}

The authors declare that there are no conflicts of interest regarding the publication of this article.

\section{Acknowledgments}

This research was funded by the National Natural Science Foundation of China (Grant nos. 11972034 and 11802213), Strategic Priority Research Program of the Chinese Academy of Sciences (Grant no. XDB22040301), and Youth Innovation Promotion Association of the Chinese Academy of Science (Grant no. 2020018).

\section{References}

[1] B. Assouar, B. Liang, Y. Wu, Y. Li, J.-C. Cheng, and Y. Jing, "Acoustic metasurfaces," Nature Reviews Materials, vol. 3, no. 12, pp. 460-472, 2018.

[2] Z. Liu, X. Zhang, Y. Mao et al., "Locally resonant sonic materials," Science, vol. 289, no. 5485, pp. 1734-1736, 2000.
[3] P. Sheng, X. X. Zhang, Z. Liu et al., "Locally resonant sonic materials," Physica B: Condensed Matter, vol. 338, no. 1-4, pp. 201-205, 2003.

[4] G. Wang, X. Wen, J. Wen et al., "Two-dimensional locally resonant phononic crystals with binary structures," Physical Review Letters, vol. 93, no. 15, Article ID 154302, 2004.

[5] Z. Liu, C. T. Chan, and P. Sheng, "Analytic model of phononic crystals with local resonances," Physical Review B, vol. 71, no. 1, Article ID 014103, 2005.

[6] J. Mei, Z. Liu, W. Wen et al., Effective Mass Density of FluidSolid Composites Physical Review Letters, vol. 96, no. 2, Article ID 024301, 2006.

[7] R. Sainidou, B. Djafari-Rouhani, Y. Pennec et al., "Locally resonant phononic crystals made of hollow spheres or cylinders," Physical Review B, vol. 73, no. 2, Article ID 024302, 2006.

[8] Z. Yang, J. Mei, M. Yang et al., Membrane-type Acoustic Metamaterial with Negative Dynamic Mass Physical Review Letters, vol. 101, no. 20, Article ID 204301, 2008.

[9] Z. Yang, H. M. Dai, N. H. Chan et al., "Acoustic metamaterial panels for sound attenuation in the $50-1000 \mathrm{~Hz}$ regime," Applied Physics Letters, vol. 96, no. 4, Article ID 041906, 2010.

[10] X. Yang, J. Yin, G. Yu, L. Peng, and N. Wang, "Acoustic superlens using Helmholtz-resonator-based metamaterials," Applied Physics Letters, vol. 107, no. 19, Article ID 193505, 2015.

[11] M. Yang, G. Ma, Z. Yang et al., "Coupled membranes with doubly negative mass density and bulk modulus," Physical Review Letters, vol. 110, no. 13, 2013.

[12] C. J. Naify, C. Chang, G. P. Mcknight et al., "Membrane-type metamaterials: transmission loss of multi-celled arrays," Journal of Applied Physics, vol. 109, no. 10, 2011.

[13] C. J. Naify, C.-M. Chang, G. Mcknight, and S. R. Nutt, "Scaling of membrane-type locally resonant acoustic metamaterial arrays," The Journal of the Acoustical Society of America, vol. 132, no. 4, pp. 2784-2792, 2012.

[14] C. J. Naify, C. Chang, G. P. Mcknight et al., "Transmission loss of membrane-type acoustic metamaterials with coaxial ring masses," Journal of Applied Physics, vol. 110, no. 12, 2011.

[15] N. Fang, D. Xi, J. Xu et al., "Ultrasonic metamaterials with negative modulus," Nature Materials, vol. 5, no. 6, pp. 452-456, 2006.

[16] X. Hu, K. M. Ho, C. T. Chan et al., "Homogenization of acoustic metamaterials of Helmholtz resonators in fluid," Physical Review B, vol. 77, no. 17, Article ID 172301, 2008.

[17] S. H. Lee, C. M. Park, Y. M. Seo, Z. G. Wang, and C. K. Kim, "Acoustic metamaterial with negative modulus," Journal of Physics: Condensed Matter, vol. 21, no. 17, Article ID 175704, 2009.

[18] S. H. Lee, C. M. Park, Y. M. Seo et al., "Composite acoustic medium with simultaneously negative density and modulus," Physical Review Letters, vol. 104, no. 5, Article ID 054301, 2010.

[19] J. Li, X. Zhou, G. L. Huang et al., "Acoustic metamaterials capable of both sound insulation and energy harvesting," Smart Materials and Structures, vol. 25, no. 4, 2016.

[20] Y. Li, B. Liang, X. Tao et al., "Acoustic focusing by coiling up space," Applied Physics Letters, vol. 101, no. 23, 2012.

[21] Z. Liang, T. Feng, S. Lok et al., Space-coiling Metamaterials with Double Negativity and Conical Dispersion Scientific Reports, vol. 3, p. 1614, 2013.

[22] Y. Li, B. Liang, X.-Y. Zou, and J.-C. Cheng, "Broadband Acoustic transmission enhancement through a structured stiff 
plate with locally resonant elements," Chinese Physics Letters, vol. 29, no. 11, Article ID 114301, 2012.

[23] J. Liu, L. Li, B. Xia, and X. Man, "Fractal labyrinthine acoustic metamaterial in planar lattices," International Journal of Solids and Structures, vol. 132-133, pp. 20-30, 2018.

[24] Y. Xie, B. I. Popa, L. Zigoneanu et al., "Measurement of a broadband negative index with space-coiling acoustic metamaterials," Physical Review Letters, vol. 110, no. 17, Article ID 175501, 2013.

[25] Y. Cheng, C. Zhou, B. G. Yuan, D. J. Wu, Q. Wei, and X. J. Liu, "Ultra-sparse metasurface for high reflection of low-frequency sound based on artificial Mie resonances," Nature Materials, vol. 14, no. 10, pp. 1013-1019, 2015.

[26] T. Frenzel, J. David Brehm, T. Bückmann et al., "Three-dimensional labyrinthine acoustic metamaterials," Applied Physics Letters, vol. 103, no. 6, Article ID 061907, 2013.

[27] X. Man, T. Liu, B. Xia, Z. Luo, L. Xie, and J. Liu, "Space-coiling fractal metamaterial with multi-bandgaps on subwavelength scale," Journal of Sound and Vibration, vol. 423, pp. 322-339, 2018.

[28] A. O. Krushynska, F. Bosia, M. Miniaci, and N. M. Pugno, "Spider web-structured labyrinthine acoustic metamaterials for low-frequency sound control," New Journal of Physics, vol. 19, no. 10, Article ID 105001, 2017.

[29] A. O. Krushynska, F. Bosia, and N. M. Pugno, "Labyrinthine acoustic metamaterials with space-coiling channels for lowfrequency sound control," Acta Acustica United with Acustica, vol. 104, no. 2, pp. 200-210, 2018.

[30] M. Miniaci, A. Krushynska, A. S. Gliozzi et al., Design and Fabrication of Bioinspired Hierarchical Dissipative Elastic Metamaterials Physical Review Applied, vol. 10, no. 2, Article ID 024012, 2018.

[31] G. Y. Song, Q. Cheng, B. Huang, H. Y. Dong, and T. J. Cui, "Broadband fractal acoustic metamaterials for low-frequency sound attenuation," Applied Physics Letters, vol. 109, no. 13, Article ID 131901, 2016.

[32] G. Y. Song, B. Huang, H. Y. Dong et al., "Broadband focusing acoustic lens based on fractal metamaterials," Scientific Reports, vol. 6, no. 1, p. 35929, 2016.

[33] X. F. Fu, G. Y. Li, M. H. Lu, G. Lu, and X. Huang, "A 3D space coiling metamaterial with isotropic negative acoustic properties," Applied Physics Letters, vol. 111, no. 25, Article ID 251904, 2017.

[34] M. Yang, S. Chen, C. Fu, and P. Sheng, "Optimal soundabsorbing structures,” Materials Horizons, vol. 4, no. 4, pp. 673-680, 2017.

[35] S. Chen, Y. Fan, Q. Fu et al., "A review of tunable acoustic metamaterials," Applied Sciences, vol. 8, no. 9, p. 1480, 2018.

[36] S. Chen, Y. Fan, F. Yang et al., "Engineering coiling-up space metasurfaces for broadband low-frequency acoustic absorption," Physica Status Solidi (RRL) Rapid Research Letters, vol. 13, no. 12, Article ID 1900426, 2019.

[37] V. Fokin, M. Ambati, C. Sun et al., "Method for retrieving effective properties of locally resonant acoustic metamaterials," Physical Review B, vol. 76, no. 14, Article ID 144302, 2007. 